\title{
Laparoscopic Treatment of a Hepatoduodenal Ligament Schwannoma With Infrared Indocyanine Green Fluorescence
}

\author{
KODAI TOMIOKA ${ }^{1}$, TAKESHI AOKI ${ }^{1 *}$, TOMOTAKE KOIZUMI ${ }^{1}$, AHMED ELEWA ${ }^{2}$, TOMOKAZU KUSANO ${ }^{1}$, \\ KAZUHIRO MATSUDA ${ }^{1}$, KOJI NOGAKI ${ }^{1}$, YOSHIHIKO TASHIRO ${ }^{1}$, YUSUKE WADA $^{1}$, TOMOKI HAKOZAKI ${ }^{1}$, \\ HIDEKI SHIBATA $^{1}$, TAKAHITO HIRAI ${ }^{1}$, TATSUYA YAMAZAKI ${ }^{1}$, KAZUHIKO SAITO ${ }^{1}$, \\ YUTA ENAMI ${ }^{1}$, IKUYA SUGIURA ${ }^{3}$, YOKO NAKAJIMA ${ }^{3}$, JUN ARAI ${ }^{3}$, ATSUSHI KAJIWARA ${ }^{3}$, \\ SHOJIRO UOZUMI ${ }^{3}$, YU SHIMOZUMA ${ }^{3}$, MANABU UCHIKOSHI ${ }^{3}$, MASASHI SAKAKI ${ }^{3}$, \\ HITOSHI YOSHIDA ${ }^{3}$, SAKIKO MIURA ${ }^{4}$ and MASAHIKO MURAKAMI ${ }^{1}$ \\ ${ }^{1}$ Division of Gastroenterological and General Surgery, Department of Surgery, Showa University, Tokyo, Japan; \\ ${ }^{2}$ National Hepatology and Tropical Medicine Research Institute, Cairo, Egypt; \\ ${ }^{3}$ Department of Medicine, Division of Gastroenterology, Showa University, Tokyo, Japan; \\ ${ }^{4}$ Department of Medicine, Division of Pathology, Showa University, Tokyo, Japan
}

\begin{abstract}
Schwannomas occurring in the hepatoduodenal ligament are extremely rare, with only four cases reported. Here, we describe a case of a 30-mm schwannoma that originated in the hepatoduodenal ligament of a 38-year-old female found during a periodic medical check-up. Magnetic resonance imaging demonstrated a tumor in the hepatoduodenal ligament. Following an ultrasound-guided microbiopsy, histological examination showed solitary fibrous tumor or schwannomas in the liver or originating from the hepatoduodenal ligament. The relationship between the tumor and associated organs was confirmed intraoperatively, and the tumor was removed safely in its entirety using indocyanine green. The postoperative histopathological examination revealed the presence of a schwannoma with typical characteristics. To our knowledge, this is the first case of hepatoduodenal ligament schwannoma treated by laparoscopic surgery using indocyanine green fluorescence imaging.
\end{abstract}

Schwannomas are neurogenic tumors originating from Schwann cells in nerve sheaths or cells in the peripheral nervous system (1). They appear in all ethnic groups, all

This article is freely accessible online.

Correspondence to: Takeshi Aoki, Division of Gastroenterological and General Surgery, Department of Surgery, Showa University, 15-8 Hatanodai, Shinagawa, Tokyo 142-8666, Japan. Tel: +81 337848541, Fax: +81 337845835, e-mail: takejp@med.showa-u.ac.jp

Key Words: Schwannoma, hepatoduodenal ligament, fluorescence, laparoscopic resection, indocyanine green. ages, and both sexes, worldwide (2). More than $90 \%$ of schwannomas are benign and composed of 5\% of benign soft-tissue neoplasms $(2,3)$. Although they occur in most parts of the body, the head, neck, and flexor surfaces of the extremities are the most commonly affected sites. Schwannomas are mostly found in patients aged 20-50 years, with no significant predominance of either sex (4). Most schwannomas show either monosomy 22 or loss of $22 \mathrm{q}$ material (5). They can arise in all anatomical sites, with the most common ones being the head, neck, and extremities (4), whereas $10 \%$ originate from multiple locations (6). They rarely occur in the retroperitoneum $(7,8)$, including the ligaments (8), bowel mesentery (9), and abdominal organs, including the gallbladder (7), pancreas (10), and liver (11). The most common treatment for schwannomas is surgery, and long-term survival can be expected in the majority of cases (1). Interestingly, schwannomas occurring in the hepatoduodenal ligament are extremely rare, with only four cases reported in the literature to date (12-15) (Table I). Patients presenting with schwannomas in the hepatoduodenal ligament have no specific symptoms; thus, the preoperative diagnosis is considerably difficult and the tumors are found incidentally.

The present report describes the case of a hepatoduodenal ligament schwannoma in a 38-year-old female patient and includes a review of the existing literature. Although surgical resection is the standard treatment for the schwannoma, when it occurs in the hepatoduodenal ligament, as seen in the present case, it may be difficult to dissect or remove the tumor without injuring or damaging the surrounding organs because of its close contact with the bile duct and other important vessels. Furthermore, it is sometimes necessary to resect surrounding organs, which may cause excessive surgical 
Table I. Available reports of schwannomas in the hepatoduodenal ligament published in the literature.

\begin{tabular}{|c|c|c|c|c|c|c|c|c|}
\hline Author & Year & Age & Gender & Country & Preoperative diagnosis & Treatment & Size $(\mathrm{mm})$ & $\begin{array}{l}\text { Follow-up } \\
\text { (Months) }\end{array}$ \\
\hline Nagafuchi et al. (12) & 1993 & 62 & Female & Japan & Mass in the hepatic hilus & $\begin{array}{l}\text { Laparotomy, } \\
\text { cholecystectomy }\end{array}$ & $90 * 50 * 45$ & 26 \\
\hline Pinto et al. (13) & 2011 & 29 & Male & Portugal & $\begin{array}{l}\text { Spindle cell neoplasia } \\
\text { or stromal tumor }\end{array}$ & Laparotomy & $45 * 29$ & NA \\
\hline Tao et al. (14) & 2016 & 50 & Male & China & Stromal tumor & Laparoscopic surgery & $45 * 25 * 25$ & 7 \\
\hline Xu et al. (15) & 2016 & 43 & Male & China & Abdominal tumor & Laparotomy & $85 * 55 * 30$ & 17 \\
\hline Present case & 2019 & 38 & Female & Japan & Spindle cell neoplasia & $\begin{array}{c}\text { Laparoscopic surgery } \\
\text { with ICG }\end{array}$ & $30 * 28 * 17$ & 14 \\
\hline
\end{tabular}

NA: Not available; ICG: indocyanine green.

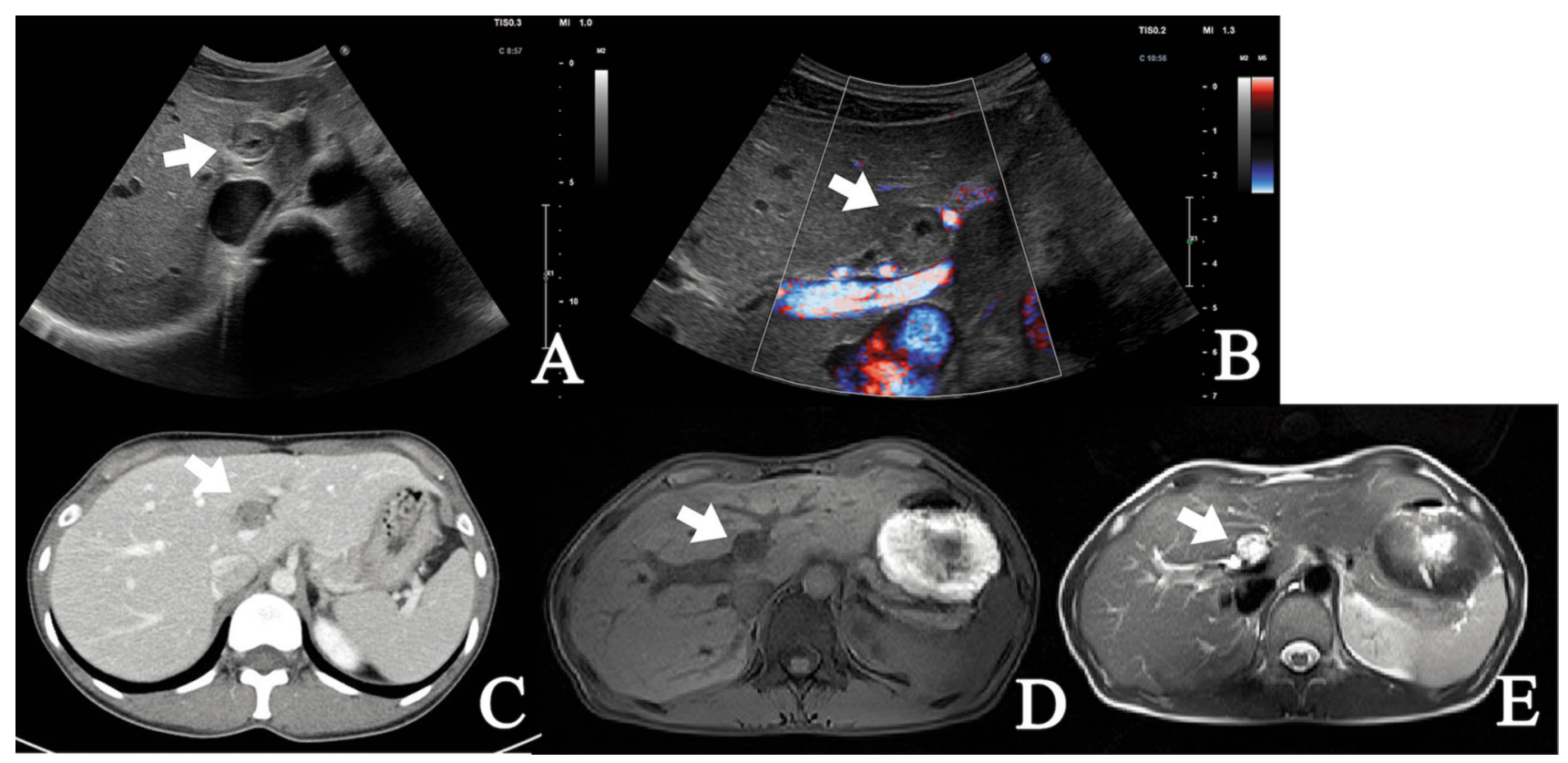

Figure 1. White arrow points to a tumor. (A) Abdominal mass detected using conventional ultrasound. (B) Color Doppler ultrasound showed no blood supply in the tumor mass. (C) Enhanced computed tomography demonstrated a hypodense tumor approximately 25 mm in diameter beside the portal vein. (D) Magnetic resonance imaging of the tumor; T1-weighted sequences. (E) Magnetic resonance imaging of the tumor; T2-weighted sequences.

stress. In the present case, the relationship between the tumor and the organs was confirmed intraoperatively, and the tumor was removed safely in its entirety using indocyanine green (ICG). ICG binds to serum proteins in vivo and expresses a fluorescent signal (16). ICG fluorescence imaging has previously clearly demarcated the liver segments of a liver tumor $(17,18)$ and is, thus, extremely useful in this case. Histopathological and immunohistological evaluation is generally needed to confirm the diagnosis of schwannomas. Two main tissue patterns are generally recognized in schwannomas, designated as: i) Antoni type A (interlacing and cellular fascicles) and ii) B tissues (less cellular and myxoid) regions (14). Immunohistochemically, schwannomas are strongly positive for S-100 and negative for desmin, smooth muscle myosin, $\alpha$-SMA, CD34, and CD117 (19).

To our knowledge, this is the first case of a patient who underwent laparoscopic surgery using infrared ICG fluorescence to visually distinguish the organs adjacent to the tumor, such as the liver, hepatic artery, portal vein, and common bile duct in the hepatic portal region, and to avoid a bile duct injury.

\section{Case Report}

A 38-year-old female with no symptoms was referred to our hospital for a detailed examination due to a liver tumor pointed 
out during a periodic medical check-up. Laboratory testing revealed normal levels for all items, with serum tumor markers, including carcinoembryonic antigen, cancer antigen (CA) 19-9, alpha-fetoprotein (AFP), and squamous cell carcinoma antigen being within a normal range. Abdominal contrast-enhanced ultrasound (CEUS) using Sonazoid (perfluorobutane microbubbles; GE Healthcare, Chicago, IL, USA) revealed well-defined, noncalcified isoechoic lesions measuring $28 \times 16 \mathrm{~mm}$ in the space between the liver and the gallbladder. No blood flow signal was found in the mass using color Doppler US (Figure 1). An enhanced abdominal computed tomography (CT) scan revealed a $20 \times 15-\mathrm{mm}$, welldefined cystic and solid round mass with a ring enhancement behind the segment 4 of the liver, in the space between the porta hepatis and the stomach (Figure 1). Magnetic resonance imaging (MRI) showed a tumor in the hepatoduodenal ligament with a low signal intensity on T1-weighted images and a high signal intensity on T2-weighted images (Figure 1). Schwannomas manifest as well-defined, hypoattenuating masses on nonenhanced CT and show peripheral enhancement with an irregular pattern on contrast-enhanced CT. Delayed peripheral enhancement until the late venous phase reflects a fibrous capsule and an internal fibrillary element on CT (20). MRI composes another useful tool for determining the nature of tumors. On MRI, schwannomas are usually presented as masses with a low signal intensity on T1-weighted images and inhomogeneous high signal intensity on T2-weighted images (21). At this point, US-guided microbiopsy was performed. Morphological findings were obtained using Hematoxylin and eosin staining of $3 \mu \mathrm{mformalin}$-fixed, paraffin-embedded sections, indicating a proliferation of nonatypical round-tospindle-shaped cells without mitoses. immunohistochemical investigation using the following antibodies: S-100 (Polyclonal Rabbit), $\alpha$-SMA (1A4), desmin (D33), $\beta$-catenin ( $\beta$-catenin1), Vimentin (V9) (Dako, Agilent technologies, Glostrup, Denmark), CD34 (Nichirei Bioscience, Tokyo, Japan), CD117 (c-kit) (Ventana, Tucson, AZ, USA) showed that vimentin was positive, while S-100, CD34, desmin, CD117, and alpha smooth muscle actin ( $\alpha$-SMA) were negative. These findings suggest a solitary fibrous tumor or schwannoma in the liver or originating from the hepatoduodenal ligament. Accordingly, laparoscopic resection using ICG fluorescence imaging was planned, and liver resection and lymphadenectomy were also planned if any malignant potential was observed intraoperatively. During surgery, $2.5 \mathrm{mg}$ of ICG (Diagnogreen; Daiichi Sankyo, Tokyo, Japan) was injected intravenously in one bolus. The PINPOINT Endoscopic Fluorescence Imaging System (Novadaq, Mississauga, ON, Canada) was used to visualize the liver and bile duct in a pseudo-color (green) in real time. Under the laparoscopic view, the tumor surrounded by a fibrous capsule was located in the hepatoduodenal ligament without invasion to the surrounding tissue and organs (Figure 2). ICG fluorescence imaging proved very useful for
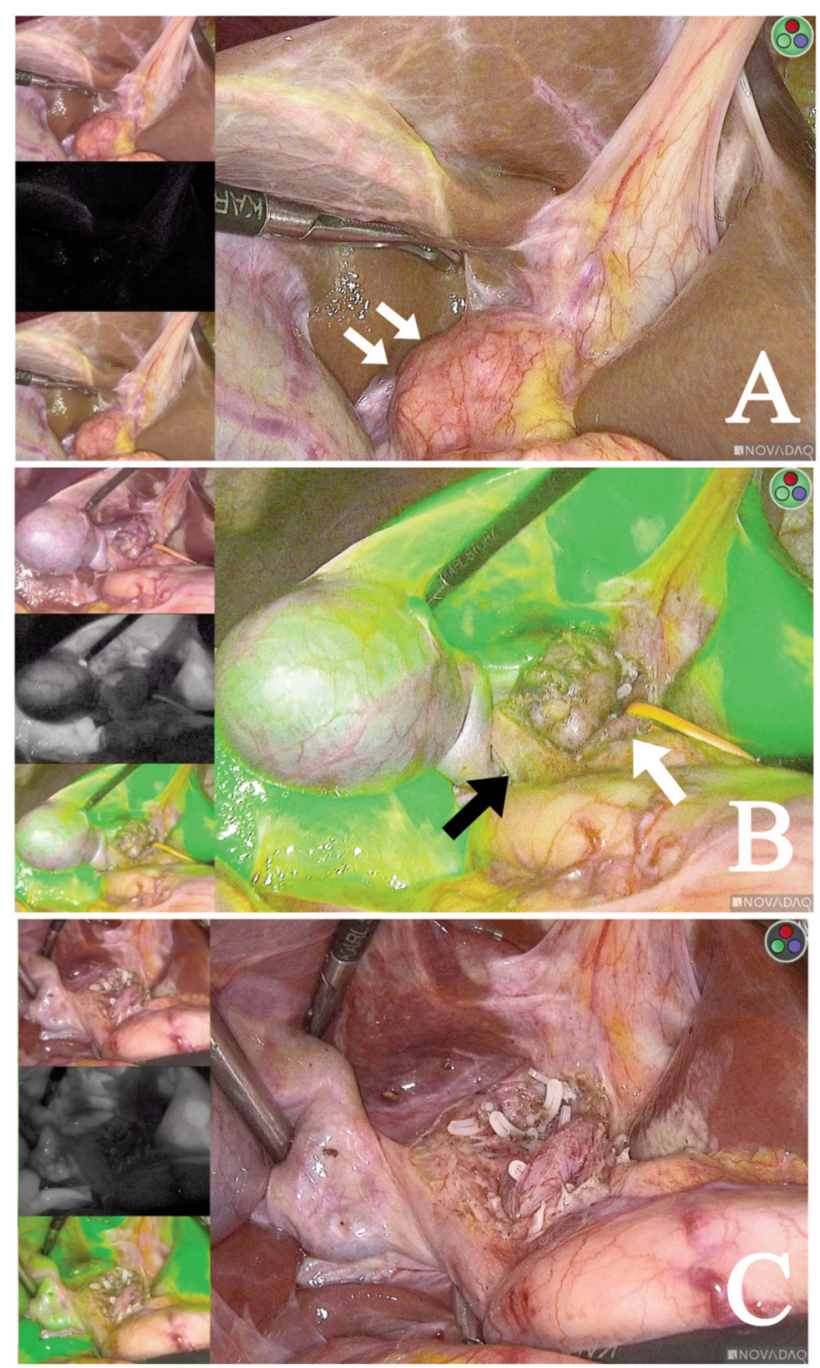

Figure 2. Intraoperative findings. The encapsulated tumor (double white arrows) was found in the hepatoduodenal ligament, with no invasion of the common hepatic artery (white arrow) (A). After ICG injection, the tumor was divided from surrounding organs (liver, hepatic artery (white arrow) and bile duct (black arrow) are presented in a pseudo-color green) (B). The tumor was completely resected $(C)$.

identifying the liver parenchyma and the common bile duct next to the tumor and confirmed the fact that the tumor did not originate in the liver, given the absence of near-infrared fluorescence (superimposed in a green pseudo-color) in the tumor. The tumor was completely resected (operation time was 208 min with a small amount of bleeding) and the gross specimen was described as a mass measuring $30 \times 28 \times 17 \mathrm{~mm}$. Both the postoperative pathological examination using hematoxylin and eosin and the immunohistochemical staining revealed that the tumor mainly consisted of: i) spindle-shaped cells with no atypia, ii) a positive expression of S-100 protein, and iii) absence of CD34, $\alpha$-SMA, desmin, and $\beta$-catenin 

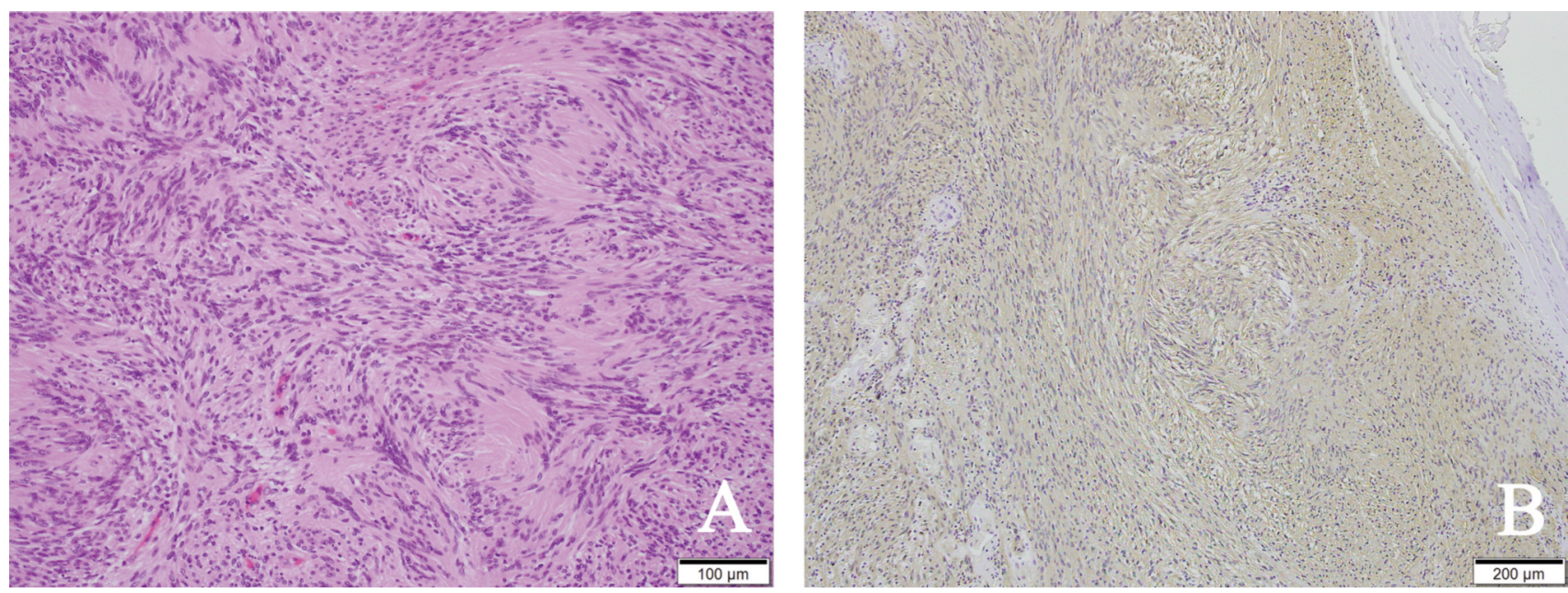

Figure 3. Tissue histology. (A) Histopathological examination revealed that the tumor was mainly composed of spindle cells (hematoxylin and eosin staining). (B) Tumor specimens were strongly positive for S-100.

expression. These results revealed the presence of a schwannoma with typical characteristics (Figure 3). In the present case, intraoperative ICG imaging and these histopathological examinations revealed that the tumor originated from the ligament rather than the liver parenchyma. The patient developed no complications after operation and was discharged on hospital day 5 .

This study was approved by a suitably constituted Ethics Committee in our institution (approval number is 1754). A written informed consent was obtained from the patient for the publication of this case report and any accompanying images.

\section{Discussion}

In the present case, the tumor occurred in the hepatoduodenal ligament, and because of its contact with the liver surface, it was quite difficult to preoperatively evaluate the site of occurrence. Although the schwannomas are benign tumors, it needs to be distinguished from a liver malignant tumor, and surgical treatment seems to be appropriate. Preoperative image evaluation should be comprehensively and carefully evaluated by CT, MRI and US, and the tumor should be diagnosed and biopsy was considered if necessary. Although schwannomas are considered benign tumors, at least histologically $(2,3)$, considering of the possibility of pressure on adjacent organs due to the tumor growth and the risk of vascular occlusion, surgical treatment to remove the tumor with its surrounding capsule is desirable. When the tumor is located in the liver edge, it may be difficult to determine whether it is primarily affiliated with the liver or an adjacent organ. Although extrahepatic tumors often have a boundary with the liver parenchyma, preoperative diagnosis can be difficult; this was true in the present case because the border was unclear. Here, while the tumor was intraoperatively thought to be benign because it was wrapped in a capsule, soft, and showed no tendency toward infiltrating the surroundings, limited minimal surgical resection was considered appropriate. A two-stage additional hepatectomy with lymphadenectomy of the hepatoduodenal ligament might be necessary when potential residual tumor cells are considered. Finally, a careful follow-up should be conducted in such cases because of the lack of evidence of a desirable surgical margin as well as a definite adjuvant chemotherapy or radiation therapy.

Regarding tumor resection at the hepatoduodenal ligament, when bile duct or vessel injury occurs, there is a concern that major hepatectomy or choledochojejunostomy might be needed as additional surgery. In this case, it was not possible to clarify whether the tumor was in the liver tumor or the mesentery preoperatively, thus the ICG fluorescence imaging helped to identify the tumor's site of origin. ICG fluorescence imaging makes it easy to distinguish between the bile duct and adjacent organs in real time, which may assist in avoiding unnecessary bile duct injury and in resecting the tumor safely with minimally invasive laparoscopic surgery. We would like to suggest that the ICG fluorescence imaging technique should be actively used in such cases.

Schwannoma in the hepatoduodenal ligament is extremely rare. We identified hepatoduodenal ligament tumor intraoperatively using ICG fluorescence imaging, we were able to avoid injuring the bile duct or performing unnecessary hepatectomy. To the best of our knowledge, this is the first reported case of benign schwannoma in the hepatoduodenal ligament treated by laparoscopic surgery using ICG fluorescence imaging. 


\section{Conflicts of Interest}

The Authors disclose that they have no significant relationships with, or financial interest in, any commercial companies pertaining to this article.

\section{Authors' Contributions}

All Authors examined the patients, IS, YN, JA, AK, SU, YS, MU, MS and HY investigated the specimens via liver needle biopsies, KT, TA, TK and MM performed the operation, KT, AE, TA, TK, TK, SM and MM discussed in graphic analysis, KT, TA, TK and MM design the study concept, KT wrote the paper. All Authors read and approved the final manuscript.

\section{Acknowledgements}

The Authors thank the patient.

\section{References}

1 Le Guellec S: Nerve sheath tumours. Ann Pathol 35(1): 54-70, 2015. PMID: 25541115. DOI: 10.1016/j.annpat.2014.11.008

2 Ariel IM: Tumors of the peripheral nervous system. CA Cancer J Clin 33(5): 282-299, 1983. PMID: 6413007. DOI: 10.3322/ canjclin.33.5.282

3 Pilavaki M, Pilavaki M, Chourmouzi D, Kiziridou A, Skordalaki A, Zarampoukas $\mathrm{T}$ and Drevelengas A: Imaging of peripheral nerve sheath tumors with pathologic correlation: pictorial review. Eur J Radiol 52(3): 229-239, 2004. PMID: 15544900. DOI: 10.1016/j.ejrad 2003.12 .001

4 Das Gupta TK and Brasfield RD: Tumors of peripheral nerve origin: benign and malignant solitary schwannomas. CA Cancer J Clin 20(4): 228-233, 1970. PMID: 4316984. DOI: 10.3322/ canjclin.20.4.228

5 JD, RS, KC and Devi NR: Pancreatic schwannoma - a rare case report. J Clin Diagn Res 8(7): FD15-16, 2014. PMID: 25177575. DOI: $10.7860 / J C D R / 2014 / 8465.4642$

6 Fenoglio L, Severini S, Cena P, Migliore E, Bracco C, Pomero F, Panzone S, Cavallero GB, Silvestri A, Brizio R and Borghi F: Common bile duct schwannoma: a case report and review of literature. World J Gastroenterol 13(8): 1275-1278, 2007. PMID: 17451214. DOI: 10.3748/wjg.v13.i8.1275

7 Liu LN, Xu HX, Zheng SG, Sun LP, Guo LH and Wu J: Solitary schwannoma of the gallbladder: a case report and literature review. World J Gastroenterol 20(21): 6685-6690, 2014. PMID: 24914396. DOI: 10.3748/wjg.v20.i21.6685

8 Bayraktutan U, Kantarci M, Ozgokce M, Aydinli B, Atamanalp SS and Sipal S. Education and Imaging. Gastrointestinal: benign cystic schwannoma localized in the gastroduodenal ligament; a rare case. J Gastroenterol Hepatol 27(5): 985, 2012. PMID: 22515807. DOI: $10.1111 / j .1440-1746.2012 .06960 . x$

9 Tang SX, Sun YH, Zhou XR and Wang J: Bowel mesentery (meso-appendix) microcystic/reticular schwannoma: case report and literature review. World J Gastroenterol 20(5): 1371-1376, 2014. PMID: 24574814. DOI: 10.3748/wjg.v20.i5.1371

10 Nishikawa T, Shimura K, Tsuyuguchi T, Kiyono S and Yokosuka O: Contrast-enhanced harmonic EUS of pancreatic schwannoma.
Gastrointest Endosc 83(2): 463-464, 2016. PMID: 26341855. DOI: $10.1016 /$ j.gie.2015.08.041

11 Xu SY, Guo H, Shen Y, Sun K, Xie HY, Zhou L, Zheng SS and Wang WL: Multiple schwannomas synchronously occurring in the porta hepatis, liver, and gallbladder: first case report. Medicine (Baltimore) 95(33): e4378, 2016. PMID: 27537565. DOI: $10.1097 / \mathrm{MD} .0000000000004378$

12 Nagafuchi Y, Mitsuo H, Takeda S, Ohsato K, Tsuneyoshi M and Enjoji M: Benign schwannoma in the hepatoduodenal ligament: report of a case. Surg Today 23(1): 68-72, 1993. PMID: 7681709. DOI: $10.1007 /$ bf00309003

13 Pinto J, Afonso M, Veloso R, Tente D, Fernandes S, Proença L, Carvalho J, Pontes JM and Fraga J: Benign schwannoma of the hepatoduodenal ligament. Endoscopy 43 Suppl 2 UCTN: E195196, 2011. PMID: 21590600. DOI: 10.1055/s-0030-1256354

14 Tao L, Xu S, Ren Z, Lu Y, Kong X, Weng X, Xie Z and Hu Z: Laparoscopic resection of benign schwannoma in the hepatoduodenal ligament: A case report and review of the literature. Oncol Lett 11(5): 3349-3353, 2016. PMID: 27123115. DOI: $10.3892 / \mathrm{ol} .2016 .4410$

$15 \mathrm{Xu}$ SY, Sun K, Xie HY, Zhou L, Zheng SS and Wang WL: Schwannoma in the hepatoduodenal ligament: A case report and literature review. World J Gastroenterol 22(46): 10260-10266, 2016. PMID: 28028376. DOI: 10.3748/wjg.v22.i46.10260

16 Tanaka E, Choi HS, Fujii H Bawendi MG and Frangioni JV: Image-guided oncologic surgery using invisible light: completed pre-clinical development for sentinel lymph node mapping. Ann Surg Oncol 13(12): 1671-1681, 2006. PMID: 17009138. DOI: 10.1245/s10434-006-9194-6

17 Aoki T, Yasuda D, Shimizu Y, Odaira M, Niiya T, Kusano T, Mitamura K, Hayashi K, Murai N, Koizumi T, Kato H, Enami Y, Miwa M and Kusano M: Image-guided liver mapping using fluorescence navigation system with indocyanine green for anatomical hepatic resection. World J Surg 32(8): 1763-1767, 2008. PMID: 18543027. DOI: 10.1007/s00268-008-9620-y

18 Aoki T, Murakami M, Koizumi T, Matsuda K, Fujimori A, Kusano T, Enami Y, Goto S, Watanabe M and Otsuka K. Determination of the surgical margin in laparoscopic liver resections using infrared indocyanine green fluorescence. Langenbecks Arch Surg 403(5): 671-680, 2018. PMID: 29915961. DOI: 10.1007/s00423-018-1685-y

19 Weiss SW, Langloss JM and Enzinger FM: Value of S-100 protein in the diagnosis of soft tissue tumors with particular reference to benign and malignant Schwann cell tumors. Lab Invest 49(3): 299-308, 1983. PMID: 6310227.

20 Ciledag N, Arda K and Aksoy M: Pancreatic schwannoma: A case report and review of the literature. Oncol Lett 8(6): 27412743, 2014. PMID: 25364457. DOI: 10.3892/ol.2014.2578

21 Rha SE, Byun JY, Jung SE, Chun HJ, Lee HG and Lee JM: Neurogenic tumors in the abdomen: tumor types and imaging characteristics. Radiographics 23(1): 29-43, 2003. PMID: 12533638. DOI: $10.1148 / \mathrm{rg} .231025050$

Received February 8, 2020

Revised March 24, 2020

Accepted April 2, 2020 